\title{
ReN VM spheroids in matrix: A neural progenitor three-dimensional in vitro model reveals DYRK1A inhibitors as potential regulators of radio-sensitivity
}

\author{
Xiao Wan ${ }^{\mathrm{a}}$, Xiaoning $\mathrm{Wu}^{\mathrm{b}}$, Mark A. Hill ${ }^{\mathrm{b}}$, Daniel V. Ebner ${ }^{\mathrm{a}, *}$ \\ a Target Discovery Institute, Nuffield Department of Medicine, University of Oxford, OX3 7FZ, Oxford, England, UK \\ ${ }^{\mathrm{b}}$ Oxford Institute of Radiation Oncology, University of Oxford, OX3 7DQ, Oxford, England, UK
}

\section{ART ICLE INFO}

\section{Article history:}

Received 9 July 2020

Accepted 28 July 2020

Available online $\mathrm{xxx}$

\section{Keywords}

Three-dimensional in vitro model

Drug candidates

DYRK1

Preclinical model

Human midbrain

Cellular image analysis

Neural progenitor cells

Neurotoxicity

\begin{abstract}
A B S T R A C T
Introduction: Pre-clinical testing of small molecules for therapeutic development across many pathologies relies on the use of in-vitro and in-vivo models. When designed and implemented well, these models serve to predict the clinical outcome as well as the toxicity of the evaluated therapies. The two-dimensional (2D) reductionist approach where cells are incubated in a mono-layer on hard plastic microtiter plates is relatively inexpensive but not physiologically relevant. In contrast, well developed and applied three dimensional (3D) in vitro models could be employed to bridge the gap between $2 \mathrm{D}$ in vitro primary screening and expensive in vivo rodent models by incorporating key features of the tissue microenvironment to explore differentiation, cortical development, cancers and various neuronal dysfunctions. These features include an extracellular matrix, co-culture, tension and perfusion and could replace several hundred rodents in the drug screening validation cascade.

Methods: Human neural progenitor cells from middle brain (ReN VM, Merck Millipore, UK) were expanded as instructed by the supplier (Merck Millipore, UK), and then seeded in 96-well low-attachment plates (Corning, UK) to form multicellular spheroids followed by adding a Matrigel layer to mimic extracellular matrix around neural stem cell niche. ReN VM cells were then differentiated via EGF and bFGF deprivation for 7 days and were imaged at day 7. Radiotherapy was mimicked via gamma-radiation at $2 \mathrm{~Gy}$ in the absence and presence of selected DYRK1A inhibitors Harmine, INDY and Leucettine 41 (L41). Cell viability was measured by AlamarBlue assay. Immunofluorescence staining was used to assess cell pluripotency marker SOX2 and differentiation marker GFAP. Results: After 7 days of differentiation, neuron early differentiation marker (GFAP, red) started to be expressed among the cells expressing neural stem cell marker SOX2 (green). Radiation treatment caused significant morphology change including the reduced viability of the spheroids. These spheroids also revealed sensitizing potential of DYRK1A inhibitors tested in this study, including Harmine, INDY and L41.

Discussion \& conclusions: Combined with the benefit of greatly reducing the issues associated with in vivo rodent models, including reducing numbers of animals used in a drug screening cascade, cost, ethics, and potential animal welfare burden, we feel the well-developed and applied 3D neural spheroid model presented in this study will provide a crucial tool to evaluate combinatorial therapies, optimal drug concentrations and treatment dosages.
\end{abstract}

(C) 2020

\section{Introduction}

Human cells have been cultured for decades as in vitro models for the preclinical testing of potential disease therapies. When cultured on a plastic surface, which is the usual case for conventional in vitro models, the phenotypes of the cells start to deviate from their original tissue, potentially yielding misleading phenotypic read-outs which fail to predict the clinical behaviour of the therapeutics they were used to test [1]. Three-dimensional models have been developed to overcome

\footnotetext{
* Corresponding author. Nuffield Department of Medicine, University of Oxford, Old Road Campus, Roosevelt Drive, Headington, Oxford, OX3 7FZ, United Kingdom.

E-mail address: daniel.ebner@ndm.ox.ac.uk (D.V. Ebner)
}

some aspects of this situation. Among all of these 3D models, multicellular spheroids artificially grown using 3D in vitro techniques such as modified surfaces, scaffolds [2], or bioengineered devices such as bioreactors [3] to support a biologically active environment, are seen as a promising type of advanced in-vitro models to initially reduce and eventually replace current conventional in-vitro and in-vivo models. Cerebral organoids are emerging tools to model neural differentiation [4], cortical development [5], brain cancers [6] and various dysfunctions such as Parkinson's [7]. The abilities of the neural stem cells to self-assemble and carry on growing and differentiation are intriguing features which allow cerebral organoids to mimick a range of three-dimensional microenvironment factors, including but not limited to biological, chemical and mechanical cues [8]. 
DYRK1A (dual-specificity tyrosine-(Y)-phosphorylation-regulated kinase $1 \mathrm{~A}$ ) is an attractive target first obtained attention by its highly expression in Down's Syndrome (DS) [9]. Clinical evidence also suggest that it is involved in other neurodegenerative pathologies such as Parkinson's $[9,10]$. It plays roles in neurogenesis $[11,12]$ and relevant retina development [13]. The possibility to target DYRK1A to treat cancer was first hinted by the report that DS patients have lower chance of getting cancer [14]. DYRK1A has also found to regulate angiogenesis, which is a vital step for cancer metastasis $[15,16]$. However in contrary to its relatively clear roles in neural development and function, the exact mechanism of how DYRK1A regulate cancer progress remains mostly unknown [14,17], with most attempts focus on the cancers with low survival rate [18]. Among these, brain tumours are one group of cancer types with highly unmet needs, and there are considerable amount of observations that DYRK1A is highly likely to be involved in this deadly type of neural malignancy [19]. Blood brain barrier (BBB) is the natural barrier for the conventional chemotherapy thus makes radiotherapy particularly important first-line choice to treat brain tumours $[19,20]$.

In our previous studies, we employed morphology analysis using 3D imaging and image analysis to evaluate $3 \mathrm{D}$ in vitro models $[21,22]$ to great advantage. However, our previous research was mostly focused on immortalized cancer cell lines. Here, for the first-time, neural progenitor cell line have been cultured on a low-attachment surface to form multicellular, differentiated spheroids. The aims of this study are to extend the classic multicellular spheroids into the neural stem cell field, to mimic the normal brain tissue which can potentially be influenced by radiotherapy regimes required to treat the adjacent tumour tissue, and to demonstrate the advantages of these spheroids over the 2D model with 3Rs (replacement, reduction and refinement) potential. In addition, to further demonstrate the application potential of this model a collection of representative DYRK1A inhibitors will be tested and the responses of the ReN spheroids regarding to morphology changes and viability will be investigated.

\section{Methods}

\subsection{Maintenance of ReN VM cells}

Tissue culture flasks or well-plates were coated with laminin diluted 1:100 in ReN maintenance medium (Merck Millipore), then the cells were thawed based on supplier's instructions (Merck Millipore) and expanded in ReN growth medium, which is the ReN maintenance medium supplemented with EGF $20 \mathrm{ng} / \mathrm{mL}$, and bFGF $20 \mathrm{ng} / \mathrm{mL}$. Before the 3D culture, all the cells were cultured in a humidified incubator at $37{ }^{\circ} \mathrm{C}$ with $5 \% \mathrm{CO}_{2}$. Once $70 \%$ confluency was reached, the ReN VM cells were passaged with accutase solution (Thermo Fisher).

\subsection{D multicellular spheroid formation and treatment}

The spheroids from ReN VM cells were formed using a protocol adapted from a previously reported method $[21,22]$. Briefly, cells were cultured on low-attachment U-bottom 96-well plates (Corning-Costar). The novelty compared with previous method is the addition of a thin-layer of Matrigel after $24 \mathrm{~h}$ of cell seeding inspired by previous reports $[21,22]$. After four days, the spheroids with self-assembled 3D structures were formed and may be treated by planned regimes as 3D in vitro models for pharmacology or toxicology. The inhibitors were present in the culture medium for 7 days and the images were taken at day 7. To mimic radiation therapy, these neural spheroids were radiated by an $\mathrm{X}$-ray radiator for a constant dose of $2 \mathrm{~Gy}$. The whole experiment design is depicted in Fig. 1(A).

\subsection{AlamarBlue assay}

Overall cell viability of the spheroids was assessed by AlamarBlue assay (Invitrogen) based on previously reported protocol adapted from the manufacturer's instructions [3,21]. Briefly, AlamarBlue solution was incubated in each well for 2.5 hours, then the fluorescence intensity at $560 \mathrm{~nm}$ excitation and $590 \mathrm{~nm}$ emission was measured using a micro-plate reader (WALLAC VICTOR2 1420 multilabel counter model, PerkinElmer, UK).

\subsection{Spheroids whole fixation and immunofluorescence staining}

ReN VM spheroids in matrix were fixed by $4 \%$ PFA for at least $15 \mathrm{~min}$ and stained using a protocol modified from previous studies. The samples were first washed using PBS - glycine (100 mM glycine in PBS) three times, then blocking buffer (10\% goat serum (Sigma), $1 \%$ goat $\mathrm{F}$ (ab')2 anti-mouse immunoglobulin G (ThermoFisher, A24514) in staining buffer (PBS supplemented with $0.2 \%$ TritonX-100, 0.1\% BSA, and $0.05 \%$ Tween 20 ) was added. The samples were then incubated with primary antibodies at $4{ }^{\circ} \mathrm{C}$ overnight or at room temperature for 1.5 hours, followed by incubation in suitable second antibodies (Human anti-rat Alexa 555 A21434, human anti-rabbit Alexa 488 A27034) at room temperature for $45 \mathrm{~min}$ and finally were mounted with diaminophenylindole (DAPI) mounting medium (Abcam, UK). Images were taken and processed on a confocal microscope (Nikon, UK).

\subsection{Image analysis and display}

For image analysis on microscopy images, ImageJ (NIH) was applied to quantify the metrics of the fluorescence images. Briefly, images were opened in the programme, the RGB images were converted into 8-bit type, then the channels were split using 'Colour - Split Channels' function under the 'image' tab. The images were then segmented using 'Adjust-Threshold' function under the 'image' tab. To create a profile of fluorescence intensity, a line was drawn using the 'straight' tool among the available drawing tools along the area of interest and the function 'Analyze $\rightarrow$ Plot Profile' was applied. For structural display of compounds binding to DYRK1A, PyMol (https://pymol.org/2/) was used by 'fetch' command followed by the relevant PDB codes from the publicly available database (https://www.rcsb.org/).

\section{Results}

Development of multicellular spheroid model formed by human neural progenitor cell line ReN VM in extracellular matrix replacement Matrigel.

Compared with the ReN VM cells simply embedded in Matrigel as in Fig. 1(B)(b), or the ones just grown on low-attachment surface as in Fig. 1(B)(a), ReN VM cells formed single 3D spheroids in each well after 4 days grown on low-attachment surface as in Fig. 1(B)(c). This was followed up by 7 days growing in a thin-layered sandwich structure of Matrigel as depicted in Fig. 1(A)(c)-(d). Shown in Fig. 1(B)(d), neurite-like outgrowth started to form on the edge of spheroids.

\subsection{ReN VM spheroids responded to gamma-radiation}

It was first noticed that these spheroids were sensitive to radiation treatment indicated by observable smaller diameters after the radiation, as shown in Fig. 2(A). Immunofluorescence staining in Fig. 2(B) shows that after 7 days of differentiation, the spheroids still expressed pluripotency marker SOX2, however with considerable cells stained positive for the astrocyte marker GFAP. Interestingly, by plotting the distribution of grayscale intensity to the distance, it was revealed that after the radiation the heterogeneity of SOX2 expression signifi- 
(a)

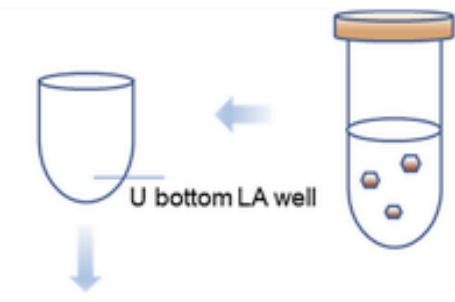

(b)

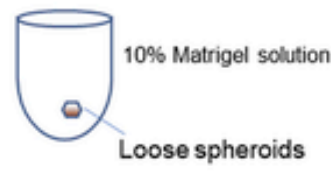

(c)

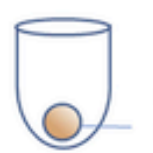

\section{Compact}

spheroids

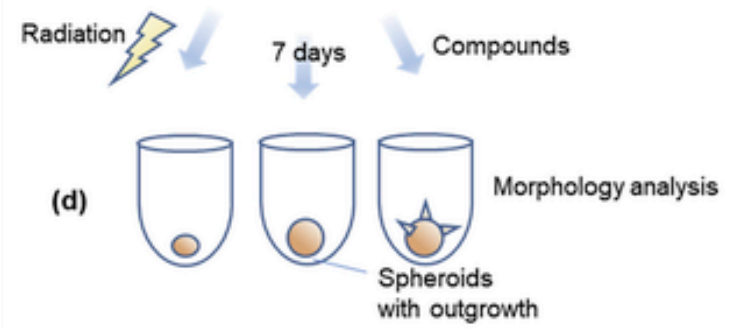

(A)
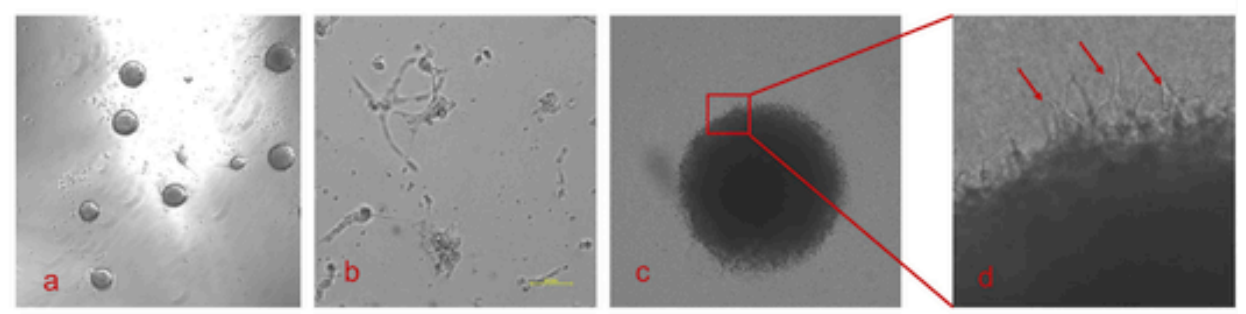

(B)

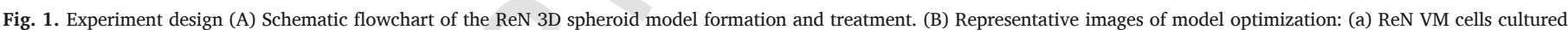

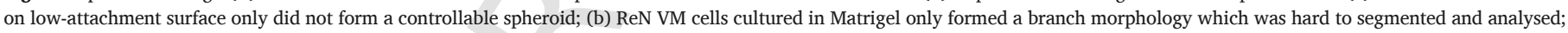

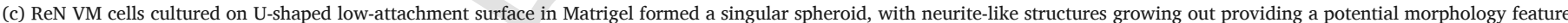

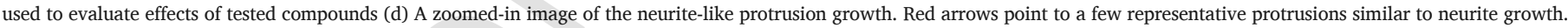
(For interpretation of the references to colour in this figure legend, the reader is referred to the Web version of this article.)

cantly increased, indicated by a more uneven pattern of the fluorescence intensity signals in Fig. 2(B). Cell viability test by AlamarBlue assay supported the fact that radiation significantly decreased the proliferative activity of these ReN VM spheroids, as shown in Fig. 2(C) ( $\mathrm{p}$-value $=0.02937$ based on Student's T-Test, $\mathrm{n}=4)$.

\subsection{DYRK1A inhibitors induced neurite-like outgrowth in ReN VM spheroids}

A brief search was done using the publicly available databased PDB to select three representative DYRK1A inhibitors Harmine, INDY and Leucettine 41 (L41) (Fig. 3(A)). A Python-based programme PyMol was applied to visualise the binding of these three inhibitors with the kinase, and the images were displayed in Fig. 3(B). AlamarBlue assay was performed with ReN VM spheroids upon 7 days treatments of $3 \mu \mathrm{M} \mathrm{L41}$, INDY and Harmine. The results suggested that, although all the DYRK1A inhibitors showed decreased overall cell viability com- pared with the control group, only Harmine had significant cytotoxicity (Fig. 3(C)) (p-value $=0.0011$ by Student's T-Test). Thus, it is interesting to observe in this study that neurite-like outgrowth formed by the treatment of INDY $3 \mu \mathrm{M}$, while no obvious outgrowth was observed in Harmine-treated group like INDY as in Fig. 4(A), although Harmine at the same concentration had the highest cytotoxicity (Fig. 3(C)) based on AlamarBlue assay.

\subsection{DYRK1A inhibitors sensitized the ReN VM spheroids to the radiation}

It was noticed that after treated by DYRK1A inhibitors, most spheroids have smaller diameters compared with the control group (Fig. 4(A)). In the presence of INDY and L41, radiation significantly decreased the viability of the ReN VM spheroids compared with the control group as shown in Fig. 4(B) (p-value $=0.0294$ for INDY, p-value $=0.005$ for L41). Comparing the INDY and L41 treated groups without and with the radiation also confirmed the efficacy of the com- 

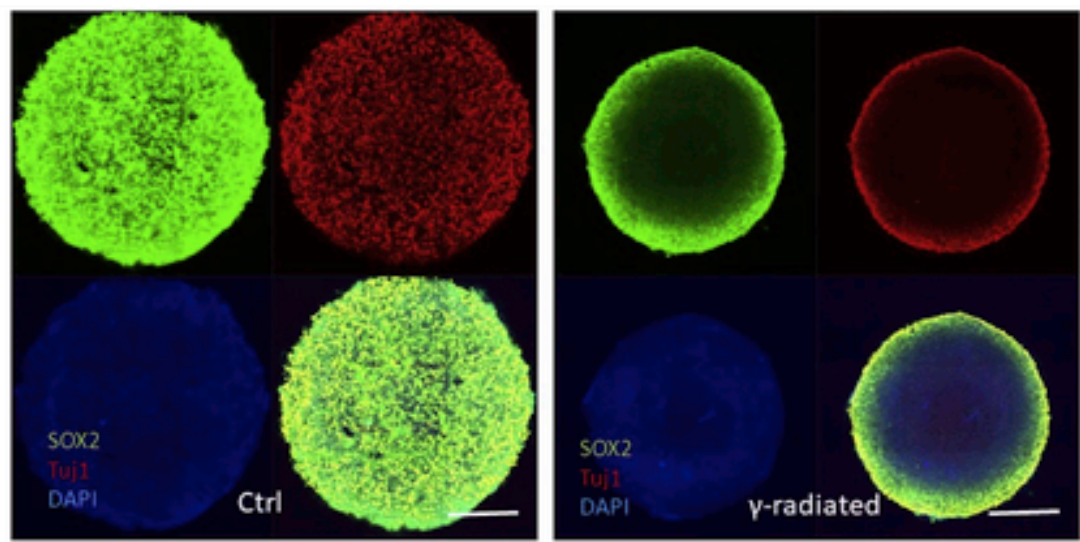

(A)
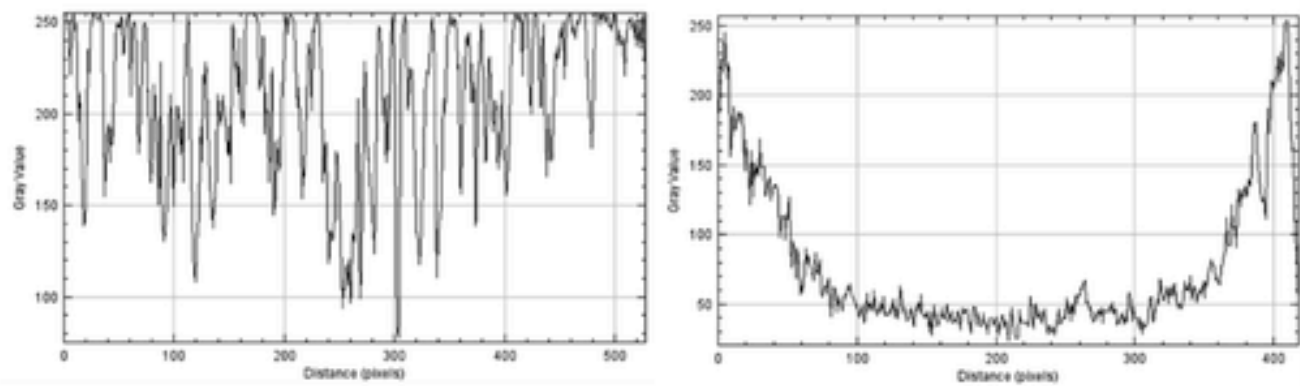

Control

Radiated

(B)

Cell Viabilitiy/AlamarBlue Fluorescence

$\square$ No radiation $=$ Radiated

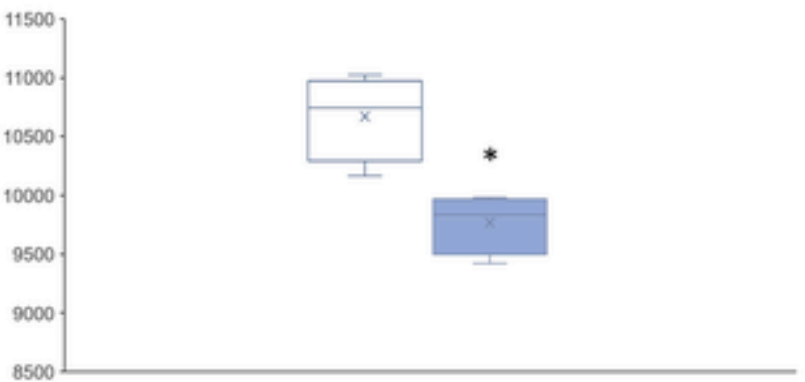

(C)

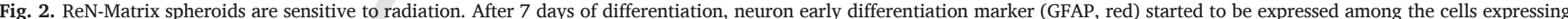

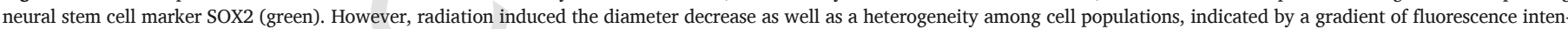

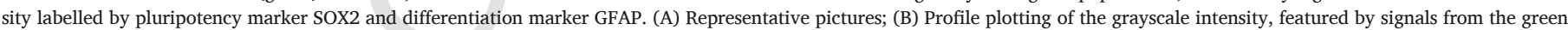

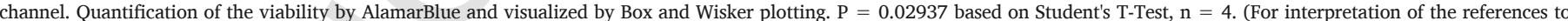
colour in this figure legend, the reader is referred to the Web version of this article.)

bination of radiation and those two DYRK1A inhibitors (p-value $=0.021$ for INDY, $\mathrm{p}$-value $=0.003$ for L41). Interestingly, the neurite-like structures were enhanced by the DYRK1A inhibitors INDY and L41, as was firstly noted in the bright-field images in Fig. 4(A). Further investigation using immunofluorescence revealed that a co-localisation pattern of progenitor marker SOX2 (green fluorescence) and astrocyte-like marker GFAP (red fluorescence), as shown in Fig. 4(C). This suggests a potential differentiated phenotype [21] and provided a possible explanation for the radio-sensitisation effects of INDY and L41 [23].

\section{Discussion}

Pre-clinical testing of therapeutics such as radiotherapy relies on the use of in-vitro and in-vivo models. These models serve to predict the clinical outcome as well as the toxicity of the evaluated therapies. It is widely acknowledged that human brain is one of the hardest to be modelled in vitro. Spheroids have been applied in this area [24]. Our previous reports demonstrated the potential of morphology analysis on the implying the maintenance of viability of the cells in the spheroids and a differentiated phenotype change [21]. Paediatric GBM is one 

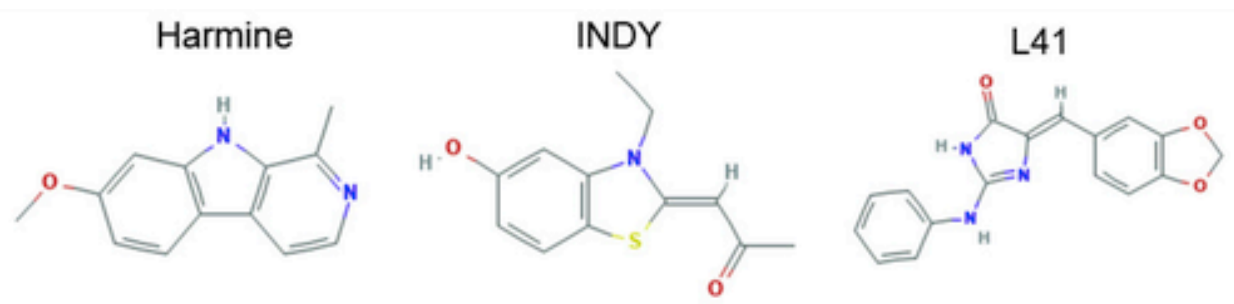

(A)
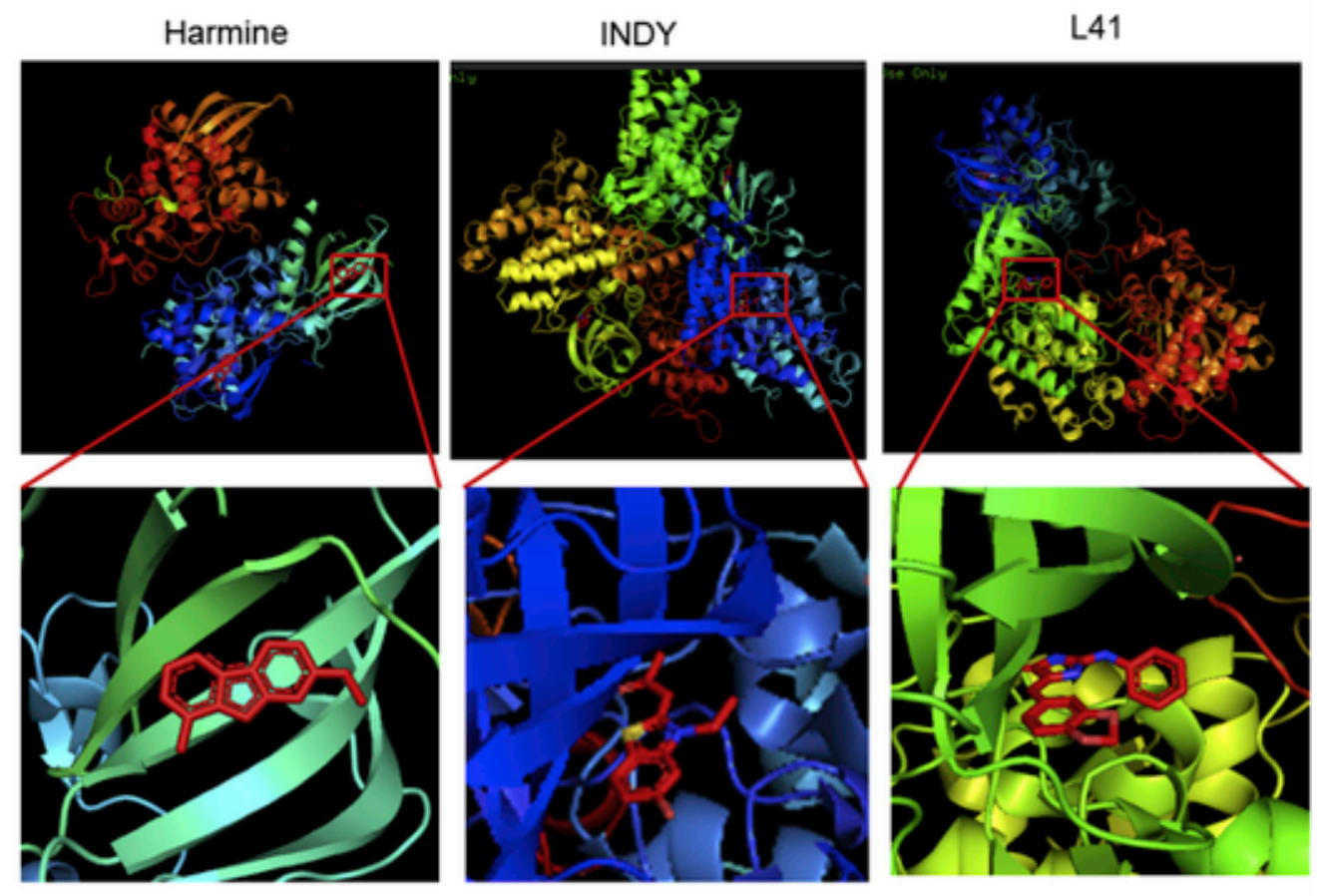

(B)

Cell Viability/AlamarBlue Fluorescence

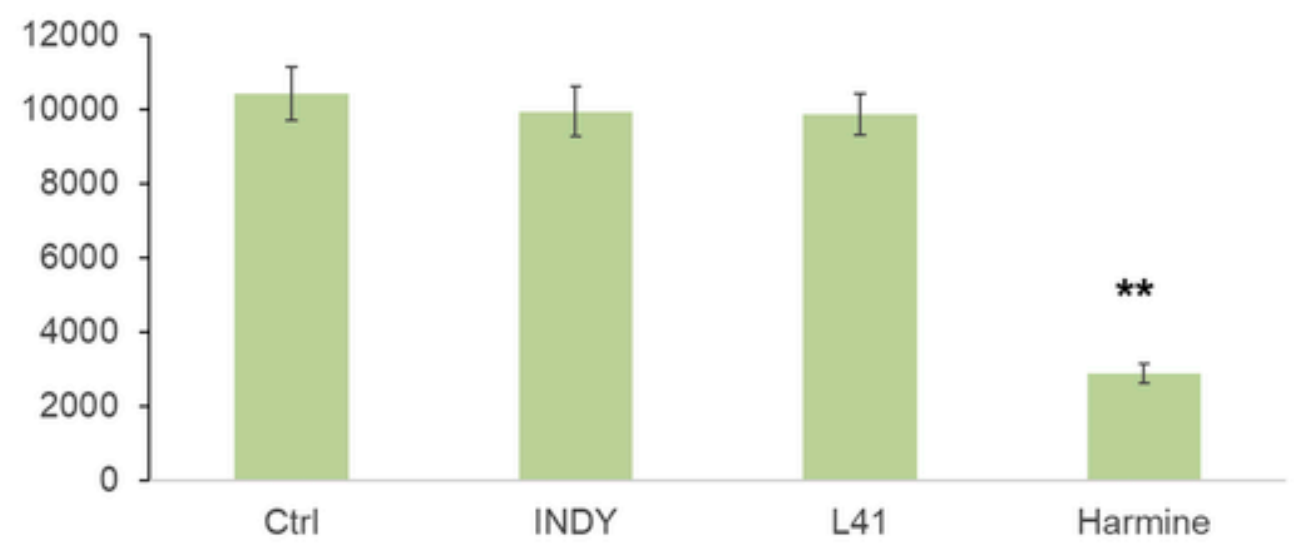

(C)

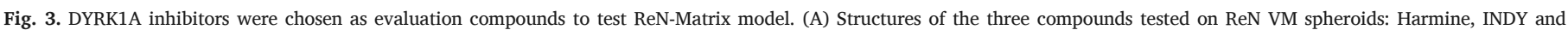

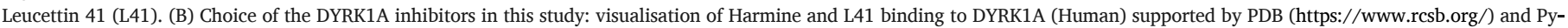




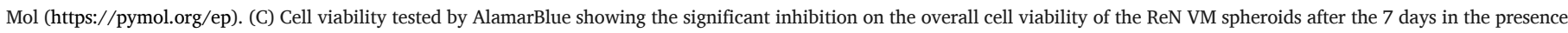
of the DYRK1A inhibitors at the concentration of $3 \mu \mathrm{M}$. ${ }^{*} \mathrm{p}<0.05,{ }^{* *}<0.01$ based on Student's T-Test.

of the most common cancer in children and radiation is a commonly used therapy for patients [25] Notably, neural stem cell niche is the most sensitive area to the toxicity caused by cancer treatment, including radiotherapy and chemotherapy, and treatment-induced damage to NSC causes cognitive defect in patients [26]. Fig. 2 in this study might support this as the radiation reduced the pluripotency marker SOX2 expression in the ReN VM cells. These models will hopefully be used not only to study neural development but also neural oncology such as GBM, as currently most knowledge in GBM obtained from animal models rather than human-derived models [25]. In addition, previous reported studies have shown that DYRK1A inhibitors have anti-cancer potential in suppressing the growth of GBM, NSCLC and PDAC [18,19,27].

DYRK1A has been studied for neural development for decades. DYRK1A inhibits cell cycle progression via regulating the expression of Cyclin D1 and cyclin-dependent kinase (CDK) inhibitor p27 [28]. DYRK1A also inhibits the proliferation of neuronal cells by phosphorylating p53, leading to the activation of CDK inhibitor p21-mediated cell cycle arrest [12]. By facilitating the assembly of dimerization partner (DP), retinoblastoma (RB)-like, E2F and MuvB (DREAM) complex, DYRK1A promots quiescence and senescence [29]. Moreover, previous studies showed that DRYK1A deregulates neuron-restrictive silencer factor (REST/NRSF) and reduces pluripotency of embryonic stem cells [30]. Other neurogenesis pathways such as Notch signalling [31] and canonical WNT pathways [32] were also interact with DYRK1A. Considerable evidence suggesting that DYRK1A inhibitor such as Harmine might induce NSC proliferation and differentiation [11,33], the neurite-induction effects observed in this study can possibly be explained by this differentiation induction capacity of DYRK1A inhibition.

The seek and choice of DYRK1A inhibitors remain intriguing topics on their own. Studies already looked into the neuroprotection potential of DYRK1A inhibitor EGCG, which is a green-tea derived compound $[34,35]$. However, later reports suggested selective DYRK1A inhibitors such as Harmine and INDY would be better options, suggesting many off-target effects of EGCG [36]. The cytotoxicity of these tested DYRK1A inhibitors, implied by reduced cell viabilities here on the 3D ReN VM spheroids demonstrated that the neuroprotective effect of well-known EGCG cannot be generalised. In addition, Harmine and INDY were reported to bind to similar domains of DYRK1A, whereas the latter has a higher selectivity [36]. Thus, it is very interesting to observe in this study the distinctive effects of Harmine and INDY on the ReN VM spheroids - Harmine significantly reduced the cell viability of the spheroids even in the absence of the radiation, while INDY only inhibited the spheroid viability after the radiation treatment. In addition, at the same concentration $(3 \mu \mathrm{M})$, INDY induced the growth of neurite-like structures whereas Harmine did not have the similar effect.

Glioma stem cells and their surrounding stroma such as vasculature have been reported to drive GBM formation and progression [37], and are highly radioresistant [20]. Future investigation might look into the potential of DRYK1A inhibition for radiosensitisation in GBM stem cells, as well as consider the co-culture with other stromal cells such as vascular endothelial cells. In conclusion, in this study: 1. A 3D in vitro model of neurotoxicity was established and 2 . The model was validated by being tested in radiation mimicking radiotherapy; 3. Application of the model to test the effects of a collection of representative DYRK1A inhibitors suggested that these compounds have the potential to sensitise the neural stem cells to radiation.

\section{Funding}

This work was partially supported by a Training Fellowship (National Centre for the Replacement, Refinement, and Reduction of Animals in Research, Grant Number NC/P002374/1).

\section{Declaration of competing interest}

The authors do not declare any conflict of interests.

\section{Acknowledgements}

Mr Steven Ball (Oxford Instruments) kindly helped with the manuscript and experiments. Dr James Thompson (Oncology, University of Oxford) kindly assisted in the radiation experiment of the neural spheroids. Dr Andrew Ball (Target Discovery Institute, University of Oxford) has kindly provided advice on the chemistry discussion of the DYRK1A inhibitors. Miss Valentina Ball warmly encouraged me through the difficulties.

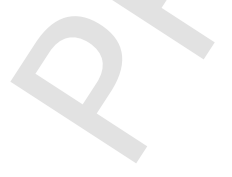

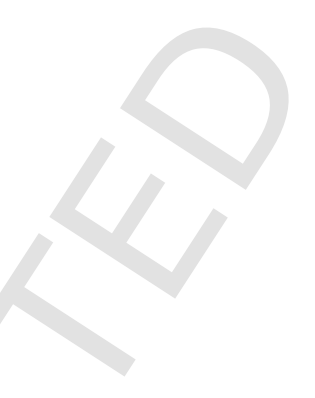




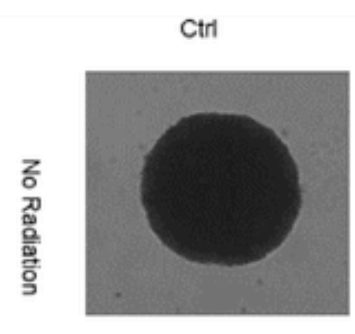

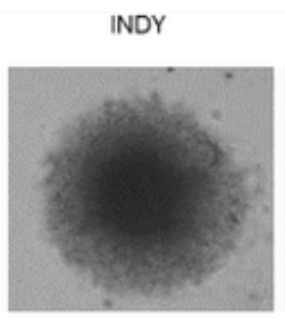

Harmine
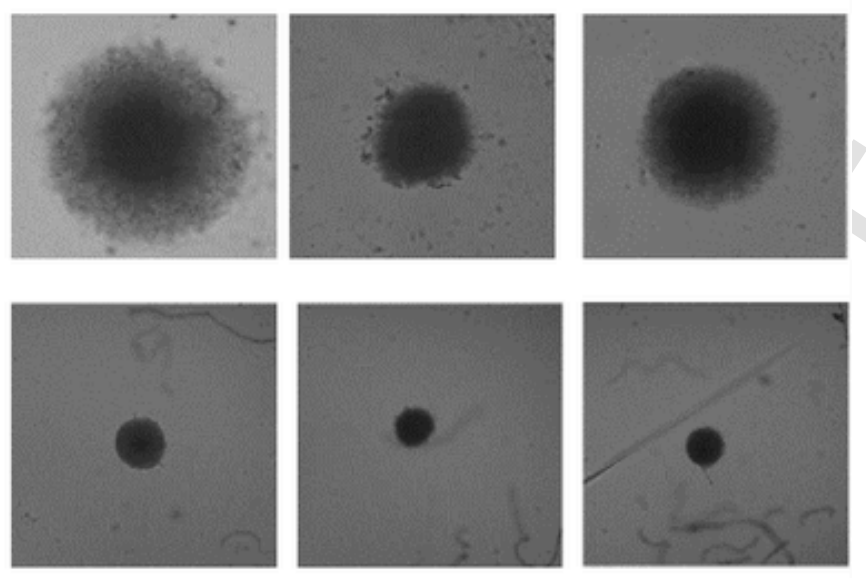

(A)

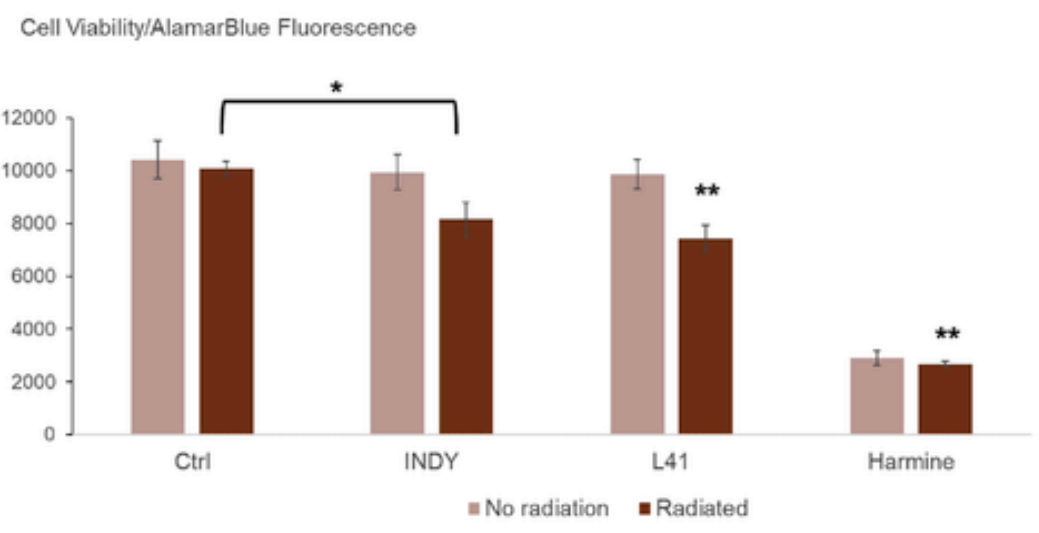

(B)
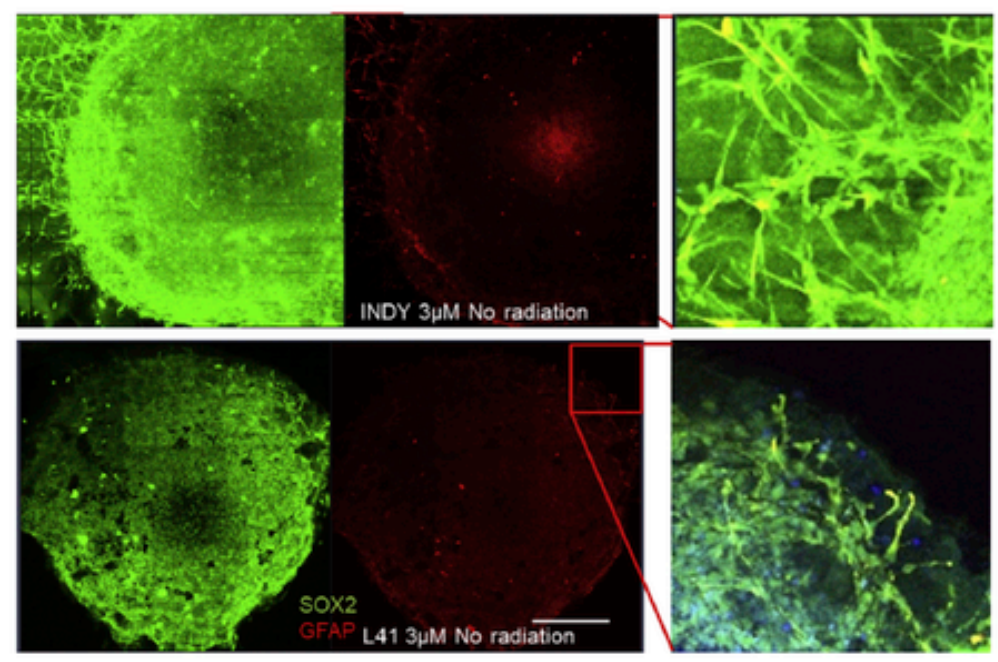

(C)

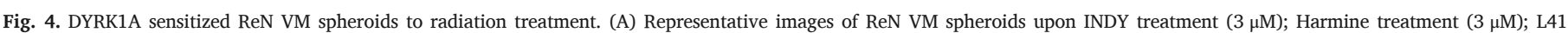

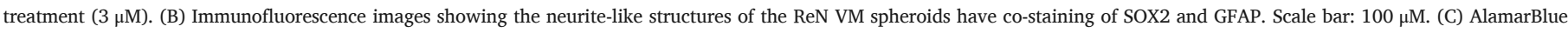
viability assay showing the addition of DYRK1A inhibitors significantly reduced the viability of ReN VM spheroids post-radiation. *p $<0.05$, ** $<0.01$ based on Student's T-Test. 


\section{References}

[1] L Song, X Yuan, Z Jones, K Griffin, Y Zhou, T Ma, Y Li, Assembly of human stem cell-derived cortical spheroids and vascular spheroids to model 3-D brain-like tissues, Sci. Rep. 9 (2019) 5977-5977.

[2] X Gong, C Lin, J Cheng, J Su, H Zhao, T Liu, X Wen, P Zhao, Generation of multicellular tumor spheroids with microwell-based agarose scaffolds for drug testing, PloS One 10 (2015) e0130348.

[3] X Wan, Z Li, H Ye, Z Cui, Three-dimensional perfused tumour spheroid model for anti-cancer drug screening, Biotechnol. Lett. 38 (2016) 1389-1395.

[4] M Eiraku, K Watanabe, M Matsuo-Takasaki, M Kawada, S Yonemura, M Matsumura, T Wataya, A Nishiyama, K Muguruma, Y Sasai, Self-organized formation of polarized cortical tissues from ESCs and its active manipulation by extrinsic signals, Cell Stem Cell 3 (2008) 519-532.

[5] M Madhavan, Z S Nevin, H E Shick, E Garrison, C Clarkson-Paredes, M Karl, B L L Clayton, D C Factor, K C Allan, L Barbar, T Jain, P Douvaras, V Fossati, R H Miller, P J Tesar, Induction of myelinating oligodendrocytes in human cortical spheroids, Nat. Methods 15 (2018) 700-706.

[6] A Linkous, D Balamatsias, M Snuderl, L Edwards, K Miyaguchi, T Milner, B Reich, L Cohen-Gould, A Storaska, Y Nakayama, E Schenkein, R Singhania, S Cirigliano, T Magdeldin, Y Lin, G Nanjangud, K Chadalavada, D Pisapia, C Liston, H A Fine, Modeling patient-derived glioblastoma with cerebral organoids, Cell Rep. 26 (2019) 3203-3211 e3205.

[7] L M Smits, L Reinhardt, P Reinhardt, M Glatza, A S Monzel, N Stanslowsky, M D Rosato-Siri, A Zanon, P M Antony, J Bellmann, S M Nicklas, K Hemmer, X Qing, E Berger, N Kalmbach, M Ehrlich, S Bolognin, A A Hicks, F Wegner, J L Sterneckert, J C Schwamborn, Modeling Parkinson's disease in midbrain-like organoids, NPJ Parkinsons Dis 5 (2019) 5.

[8] Y T Dingle, M E Boutin, A M Chirila, L L Livi, N R Labriola, L M Jakubek, J R Morgan, E M Darling, J A Kauer, D Hoffman-Kim, Three-dimensional neural spheroid culture: an in vitro model for cortical studies, tissue engineering, Part C, Methods 21 (2015) 1274-1283.

[9] J Wegiel, C-X Gong, Y-W Hwang, The role of DYRK1A in neurodegenerative diseases, FEBS J. 278 (2011) 236-245.

[10] M J Barallobre, C Perier, J Bové, A Laguna, J M Delabar, M Vila, M L Arbonés, DYRK1A promotes dopaminergic neuron survival in the developing brain and in a mouse model of Parkinson's disease, Cell Death Dis. 5 (2014) e1289-e1289.

[11] A Nakano-Kobayashi, T Awaya, I Kii, Y Sumida, Y Okuno, S Yoshida, T Sumida, H Inoue, T Hosoya, M Hagiwara, Prenatal neurogenesis induction therapy normalizes brain structure and function in Down syndrome mice, Proc. Natl. Acad. Sci. U.S.A. 114 (2017) 10268-10273.

[12] J Park, Y Oh, L Yoo, M S Jung, W J Song, S H Lee, H Seo, K C Chung, Dyrk1A phosphorylates p53 and inhibits proliferation of embryonic neuronal cells, J. Biol. Chem. 285 (2010) 31895-31906.

[13] A Laguna, S Aranda, M J Barallobre, R Barhoum, E Fernández, V Fotaki, J M Delabar, S de la Luna, P de la Villa, M L Arbonés, The protein kinase DYRK1A regulates caspase-9-mediated apoptosis during retina development, Dev. Cell 15 (2008) 841-853.

[14] Y Birger, S Izraeli, DYRK1A in Down syndrome: an oncogene or tumor suppressor?, J. Clin. Invest. 122 (2012) 807-810.

[15] H-J Cho, J-G Lee, J-H Kim, S-Y Kim, Y H Huh, H-J Kim, K-S Lee, K Yu, J-S Lee, Vascular defects of DYRK1A knockouts are ameliorated by modulating calcium signaling in zebrafish, Disease Models \& Mechanisms 12 (2019) dmm037044.

[16] E J Rozen, J Roewenstrunk, M J Barallobre, C Di Vona, C Jung, A F Figueiredo, J Luna, C Fillat, M L Arbonés, M Graupera, M A Valverde, S de la Luna, DYRK1A kinase positively regulates angiogenic responses in endothelial cells, Cell Rep. 23 (2018) 1867-1878.

[17] R Abbassi, T G Johns, M Kassiou, L Munoz, DYRK1A in neurodegeneration and cancer: molecular basis and clinical implications, Pharmacol. Therapeut. 151 (2015) 87-98.

[18] J Luna, J Boni, M Cuatrecasas, X Bofill-De Ros, E Núñez-Manchón, M Gironella, E C Vaquero, M L Arbones, S de la Luna, C Fillat, DYRK1A modulates c-MET in pancreatic ductal adenocarcinoma to drive tumour growth, Gut 68 (2019) 1465-1476.

[19] N Pozo, C Zahonero, P Fernández, J M Liñares, A Ayuso, M Hagiwara, A Pérez, J R Ricoy, A Hernández-Laín, J M Sepúlveda, P Sánchez-Gómez, Inhibition of DYRK1A destabilizes EGFR and reduces EGFR-dependent glioblastoma growth, J. Clin. Invest. 123 (2013) 2475-2487.

[20] S Bao, Q Wu, R E McLendon, Y Hao, Q Shi, A B Hjelmeland, M W Dewhirst, D D Bigner, J N Rich, Glioma stem cells promote radioresistance by preferential activation of the DNA damage response, Nature 444 (2006) 756-760.

[21] X Wan, S Ball, F Willenbrock, S Yeh, N Vlahov, D Koennig, M Green, G Brown, S Jeyaretna, Z Li, Z Cui, H Ye, E O'Neill, Perfused three-dimensional organotypic culture of human cancer cells for therapeutic evaluation, Sci. Rep. 7 (2017) 9408

[22] X Wan, P Bovornchutichai, Z Cui, E O'Neill, H Ye, Morphological analysis of human umbilical vein endothelial cells co-cultured with ovarian cancer cells in 3D: an oncogenic angiogenesis assay, PloS One 12 (2017) e0180296-e0180296.

[23] O A Mineyeva, D V Bezriadnov, A V Kedrov, A A Lazutkin, K V Anokhin, G N Enikolopov, Radiation induces distinct changes in defined subpopulations of neural stem and progenitor cells in the adult Hippocampus, Front. Neurosci. 12 (2019) 1013-1013.

[24] P Zhuang, A X Sun, J An, C K Chua, S Y Chew, 3D neural tissue models: from spheroids to bioprinting, Biomaterials 154 (2018) 113-133.

[25] D Sturm, S M Pfister, D T W Jones, Pediatric gliomas: current concepts on diagnosis, biology, and clinical management, J. Clin. Oncol. : official journal of the American Society of Clinical Oncology 35 (2017) 2370-2377.

[26] A Michaelidesová, J Konířová, P Bartůněk, M Zíková, Effects of radiation therapy on neural stem cells, Genes 10 (2019) 640.
[27] Y-1 Li, K Ding, X Hu, L-w Wu, D-m Zhou, M-j Rao, N-m Lin, C Zhang, DYRK1A inhibition suppresses STAT3/EGFR/Met signalling and sensitizes EGFR wild-type NSCLC cells to AZD9291, J. Cell Mol. Med. 23 (2019) 7427-7437.

[28] U Soppa, J Schumacher, V Florencio Ortiz, T Pasqualon, F J Tejedor, W Becker, The Down syndrome-related protein kinase DYRK1A phosphorylates p27(Kip1) and Cyclin D1 and induces cell cycle exit and neuronal differentiation, Cell Cycle 13 (2014) 2084-2100.

[29] L Litovchick, L A Florens, S K Swanson, M P Washburn, J A DeCaprio, DYRK1A protein kinase promotes quiescence and senescence through DREAM complex assembly, Genes Dev. 25 (2011) 801-813.

[30] C Canzonetta, C Mulligan, S Deutsch, S Ruf, A O’Doherty, R Lyle, C Borel, N Lin-Marq, F Delom, J Groet, F Schnappauf, S De Vita, S Averill, J V Priestley, J E Martin, J Shipley, G Denyer, C J Epstein, C Fillat, X Estivill, V L Tybulewicz, E M Fisher, S E Antonarakis, D Nizetic, DYRK1A-dosage imbalance perturbs NRSF/REST levels, deregulating pluripotency and embryonic stem cell fate in Down syndrome, Am. J. Hum. Genet. 83 (2008) 388-400.

[31] J Fernandez-Martinez, E M Vela, M Tora-Ponsioen, O H Ocaña, M A Nieto, J Galceran, Attenuation of Notch signalling by the down-syndrome-associated kinase DYRK1A, J. Cell Sci. 122 (2009) 1574-1583.

[32] J Y Hong, J I Park, M Lee, W A Muñoz, R K Miller, H Ji, D Gu, J Ezan, S Y Sokol, P D McCrea, Down's-syndrome-related kinase Dyrk1A modulates the p120-catenin-Kaiso trajectory of the Wnt signaling pathway, J. Cell Sci. 125 (2012) 561-569.

[33] N Göckler, G Jofre, C Papadopoulos, U Soppa, F J Tejedor, W Becker, Harmine specifically inhibits protein kinase DYRK1A and interferes with neurite formation, FEBS J. 276 (2009) 6324-6337.

[34] N A Singh, A K A Mandal, Z A Khan, Potential neuroprotective properties of epigallocatechin-3-gallate (EGCG), Nutr. J. 15 (2016) 60-60.

[35] J-B Liu, L Zhou, Y-Z Wang, X Wang, Y Zhou, W-Z Ho, J-L Li, Neuroprotective activity of epigallocatechin gallate against lipopolysaccharide-mediated cytotoxicity, Journal of Immunology Research (2016) 20164962351.

[36] Y Ogawa, Y Nonaka, T Goto, E Ohnishi, T Hiramatsu, I Kii, M Yoshida, T Ikura, H Onogi, H Shibuya, T Hosoya, N Ito, M Hagiwara, Development of a novel selective inhibitor of the Down syndrome-related kinase Dyrk1A, Nat. Commun. 1 (2010) 86.

[37] L J Brooks, S Parrinello, Vascular regulation of glioma stem-like cells: a balancing act, Curr. Opin. Neurobiol. 47 (2017) 8-15. 